\title{
Visualizing Transmissive Mutant Huntingtin by Correlative Light and Electron Microscopy and Cryo-electron Tomography
}

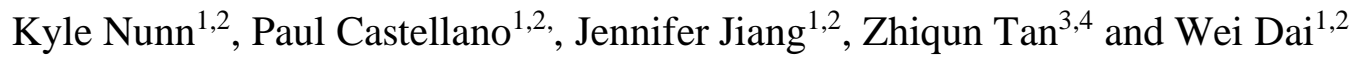

${ }^{1}$ Department of Cell Biology and Neuroscience, Rutgers, The State University of New Jersey, Piscataway, New Jersey, United States, ${ }^{2}$ Institute for Quantitative Biomedicine, Rutgers, The State University of New Jersey, Piscataway, New Jersey, United States, ${ }^{3}$ Department of Anatomy and Neurobiology, University of California - Irvine School of Medicine, Irvine, California, United States, ${ }^{4}$ Institute for Memory Impairment and Neurological Disorders, University of California - Irvine School of Medicine, Irvine, California, United States

Huntington's Disease (HD) is an inherited neurodegenerative disease caused by the abnormal expansion of a polyglutamine (polyQ) tract in the exon1 of the huntingtin (HTT) protein. The presence of the mutant HTT (mHTT) aggregates in affected cells is a hallmark of disease pathology. Recent studies have shown that mHTT can be released into the extracellular space, where they are internalized by nearby cells and thereby propagate misfolded protein pathology to surrounding cells [1-3]. However, since misfolded mHTT proteins are conventionally characterized as intracellular proteins [4-6], the structural and biochemical properties of these transmissive entities, and the molecular mechanisms of mHTT transmission remain elusive in neuroscience.

In this study, we use the conditioned medium from 14A2.6 PC-12 cells as a model system [7, 8], and applied correlative light and electron microscope (CLEM) and cryo-electron tomography (cryo-ET) to study the 3D structure of transmissive mHTT proteins. When induced by ponasterone A, these PC-12 cells express the truncated exon1 of mHTT (containing a tract of 97 glutamines, Q97) labeled by an enhanced green fluorescence protein (EGFP) at the C-terminus (mHTTex1Q97-EGFP). We observed two types of microscopic mHTTex1Q97-EGFP species in the conditioned medium from induced PC12 cells: bright puncta with clear boundary, and dim granules of various sizes without defined edges (Figure 1a).

Tomograms of the brighter puncta revealed densely packed assemblies within extracellular vesicles (EVs) (Figure 1b-d). The sizes of the EVs suggest that they are plasma membrane - originated microvesicles enclosing mHTTex1Q97-EGFP densities. The dim granules consisted of an uncoated meshwork of densities that is consistent in appearance with assemblies of mHTT oligomers (Figure 2e-g). In tomograms of uncoated mHTTex1Q97-EGFP granules, we also observed a large number of EVs surrounding these assemblies. Since the concentration of mHTTex1Q97-EGFP protein in the conditioned medium is usually too low for mHTT proteins to spontaneously form large assemblies, the co-localization of EVs and assemblies of mHTTex1Q97-EGFP meshwork leads to the postulation that EVs may modulate and promote the formation of the uncoated transmissive mHTT assemblies.

Studies on mHTT secretion mechanisms revealed that mHTT proteins can exit cells via unconventional late endosomal and lysosomal secretory pathway in a free, non-vesicular form, or through exosome- or microvesicle-mediated transport in the EV-enclosed form [3, 9]. The two types of transmissive mHTTex1Q97-EGFP species observed in the conditioned medium from induced PC-12 cells may represent aggregates following different secretion pathways. The biochemical and biophysical properties of these large assemblies, and the functional roles of EVs in modulating their formation and seeding efficiency should be explored by further experiments. 
Acknowledgements: This work was supported by the National Science Foundation (MCB-2046180) and Rutgers Busch Biomedical Research Grant to W.D. Tomographic data was collected at Rutgers CryoEM \& Nanoimaging Facility. We thank Jason Kaelber and Emre Firlar for their support in data collection.

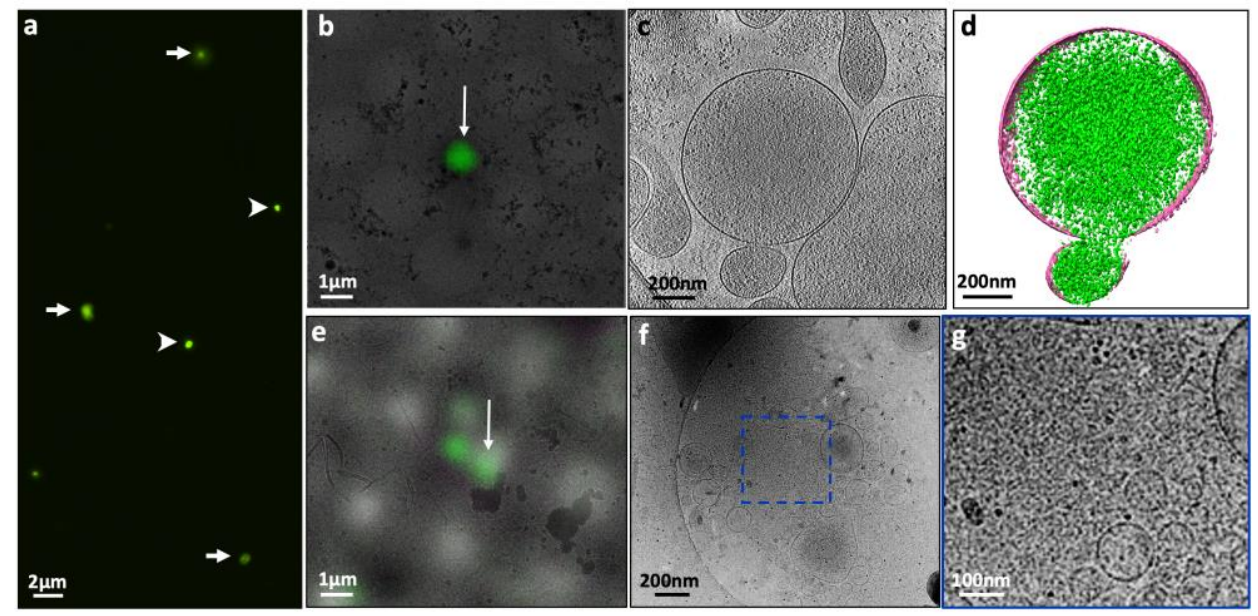

Figure 1. 3D structure of transmissive mHTT proteins revealed by CLEM and cryo-ET. (a) Fluorescent light microscopy image of the two types of mHTTex1Q97-EGFP granules in the conditioned medium from induced PC12 cells. Arrow: bright, dense puncta; Arrowhead: dim granules without clear edges. (bd) CLEM and cryo-ET of bright, dense puncta. (b) Correlative light microscopy and low magnification electron microscopy (EM) images of a representative bright punctum (long arrow). (c) EM image of the mHTTex1Q97-EGFP granule in (b) taken at 15,000x magnification. (d) volume annotation of an EV that encloses mHTTex1Q97-EGFP densities (Pink: EV membrane; Green: mHTTex1Q97-EGFP densities). (e-g) CLEM and cryo-ET of dim mHTTex1Q97-EGFP granules. (e) Correlative light microscopy and low magnification EM image of a representative diffuse granule (long arrow). (f) EM image of the mHTTex1Q97-EGFP granule in (e) taken at 15,000x magnification. (g) Zoom-in view of boxed region in (f).

\section{References}

[1] Jeon, I., et al., Acta Neuropathol, 2016. 132: p. 577-592.

[2] Masnata, M. and F. Cicchetti, Front Neurosci, 2017. 11: p. 647.

[3] Trajkovic, K., H. Jeong, and D. Krainc, Bio Protoc, 2018. 8(1): p. e2675.

[4] Bäuerlein, F.J.B., et al., Cell, 2017. 171: p. 1-9.

[5] Gruber, A., et al., Proc Natl Acad Sci U S A, 2018. 115(15): p. E3446-E3453.

[6] Peskett, T.R., et al., Mol Cell, 2018. 70(4): p. 588-601 e6.

[7] Apostol, B.L., et al., Proc Natl Acad Sci U S A, 2003. 100(10): p. 5950-5.

[8] Tan, Z., et al., Mol Psychiatry, 2015. 20(11): p. 1286-93.

[9] Trajkovic, K., H. Jeong, and D. Krainc, J Neurosci, 2017. 37(37): p. 9000-9012. 